"C2020 IEEE. Personal use of this material is permitted. Permission from IEEE must be obtained for all other uses, in any current or future media, including reprinting/republishing this material for advertising or promotional purposes, creating new collective works, for resale or redistribution to servers or lists, or reuse of any copyrighted component of this work in other works." 


\title{
High Directivity, Omnidirectional Horizontally Polarized Antenna Array for Wireless Power Transfer in Internet-of-Things Applications
}

\author{
Wei Lin and Richard W. Ziolkowski \\ Global Big Data Technologies Centre, \\ School of Electrical and Data Engineering, \\ University of Technology Sydney, \\ Ultimo 2007, Sydney, Australia, \\ Email:Wei.Lin@uts.edu.au, Richard.Ziolkowski@uts.edu.au
}

\begin{abstract}
A high directivity, compact, omnidirectional horizontally polarized (OHP) antenna array is developed for wirelessly powering internet-of-things (IoT) devices. The antenna array is realized by seamlessly inserting several phase inverters inside an electrically long $\mathbf{T E}_{0.5,0}$ mode open waveguide. The phase inverter consists of a meandered slot and eight shorting vias. The meandered slot creates an interdigitated structure on the top surface of the waveguide; it introduces capacitance. The eight shorting vias are placed in an alternating pattern on the two sides of the slot; they produce inductance. The combination of the slot and vias forms a bandpass effect and inverts the electric fields in the waveguide. Consequently, a collinear and in-phase magnetic dipole array is realized. A compact eight-element OHP magnetic dipole array is designed, fabricated and measured. The measured results confirm the design concept and high directivity $(10.4 \mathrm{dBi})$, omnidirectional HP radiation pattern has been achieved.
\end{abstract}

\section{INTRODUCTION}

Omnidirectional antennas are widely used in modern wireless communication systems due to their large radiation coverage over a set of $360^{\circ}$ directions [1] - [4]. In particular, omnidirectional horizontally polarized (OHP) antennas are becoming more and more attractive as a polarization diversity alternative to vertically polarized antennas [5] - [7]. Moreover, it has been proved that the use of the horizontally polarized antennas achieves higher reception power levels than those provided by vertically polarized systems [8]

A specific application of a high directivity OHP antenna system is illustrated in Fig. 1. It is an ideal candidate for wirelessly powering internet-of-things (IoT) devices, e.g., IoT sensors for forest fire detection and warning applications. These wirelessly powered (WP) sensors will measure data such as temperature, humidity, and smoke density, and will send this information to a gateway for processing. The WP function facilitates battery-less operation in difficult to reach or even inaccessible locations. To achieve their WP capability, such sensors need to be integrated with rectennas [9] - [12], i.e., rectifying antennas that convert incident RF power into DC power. A high directivity, efficient OHP antenna is thus highly desired to power such WP-based sensor networks.
Although many high directivity OHP antennas have been reported to date [13] - [18], it remains very challenging to achieve high directivity, compact size, simple fabrication process, and sufficient bandwidth all at the same time. This paper introduces an OHP antenna array that achieves all of these desired features at the first time. Consequently, it is the ideal source for wireless powering IoT sensors in any of the $360^{\circ}$ directions surrounding it.

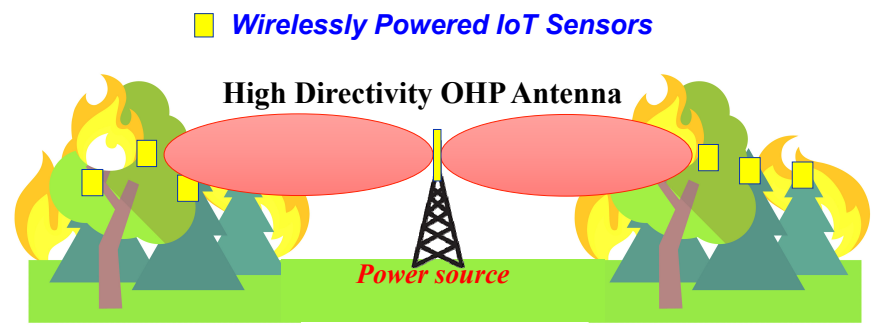

Figure 1. Illustrated IoT sensor system that is wirelessly powered by a high directivity, OHP antenna for forest fire detection and warning applications.

\section{Design, Operating Principles AND Measured Results OF THE High DiRECTIVITY, OHP ANTENNA ARRAY}

\section{A. Configuration of the antenna array}

The configuration of the OHP antenna array is shown in Fig. 2. It consists of two Rogers ${ }^{\mathrm{TM}} 5800$ substrates whose relative permittivity and loss tangent are 2.2 and 0.0009 , respectively. Substrate\#1 has a thickness of $1.575 \mathrm{~mm}$. It fills two $\mathrm{TM}_{0.5,0}$ mode open waveguides into which four phase inverters are integrated. The two center-fed waveguides are realized with substrate-integrated-waveguide (SIW) technology. Each phase inverter is a combination of a meandered slot and eight shorting vias distributed in an alternating pattern on both sides of the slot. The dimension of Sub\#l and the phase inverters are $L=137 \mathrm{~mm}, W=6.8 \mathrm{~mm}, l_{s 1}=2.9 \mathrm{~mm}, l_{s 2}=0.85 \mathrm{~mm}$, and $w_{s}$ $=0.1 \mathrm{~mm}$. Substrate\#2 is attached beneath Sub\#1 and has a thickness of $0.508 \mathrm{~mm}$. A microstrip feedline (1 to 2 power

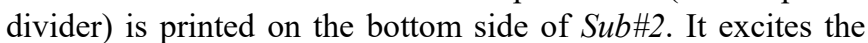
both of the $\mathrm{TM}_{0.5,0}$ mode SIW-based waveguides through two 
shorting probes in the middle. Several tuning stubs are adopted to achieve good impedance matching to a $50-\Omega$ source. The entire structure is compact and easy to fabricate. Its total volume is only $0.07 \times 0.22 \times 4.3 \lambda_{0}{ }^{3}$.

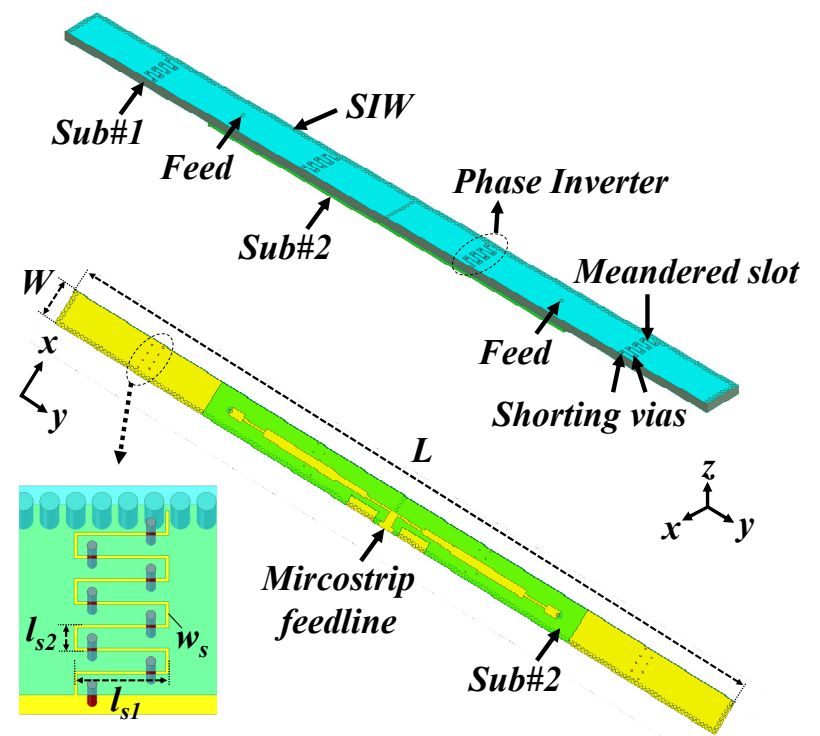

Figure 2. Configuration of the high directivity, omnidirectional, horizontally polarized antenna array.

\section{B. Operating principles}

The essence of this design is the realization of eight collinear and in-phase magnetic dipoles. As illustrated in Fig. 3, the Efield distributions along the open side of the waveguides without the phase inverters indicate that there are eight halfwavelength sections. Each section forms a magnetic dipole. Without the inverters, four of these magnetic dipoles have a reversed phase as shown. Consequently, the resulting radiation pattern is split (has a null) in the omni-plane (zx, $\varphi=0^{\circ}$ plane). The peak gain is only $7.5 \mathrm{dBi}$.
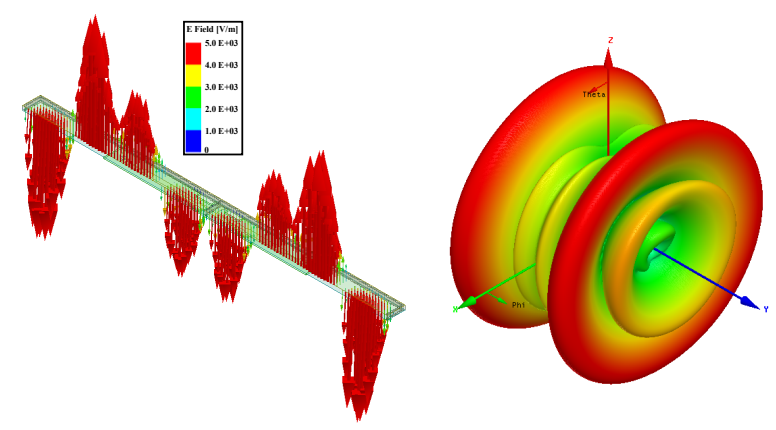

Figure 3. E-field distribution on the $\mathrm{TE}_{0.5,0}$ waveguide mode without the phase inverters and the $3 \mathrm{D}$ radiation pattern it produces at $10 \mathrm{GHz}$.

The four phase inverters are integrated seamlessly into the waveguides to achieve the same phase for all eight magnetic dipoles. The meandered slot of each phase inverter creates an interdigitated structure on the top surface of the waveguide. It introduces a capacitance. The eight shorting vias placed in the alternating pattern on both sides of the slot produce an inductance. The combination of the slot and vias forms a bandpass filter effect and inverts the electric fields of the waveguide, i.e, flips the direction of those fields by $180^{\circ}$. The electric field distribution in the structure is depicted in Fig. 4 (a) during one period of the source frequency. The effectiveness of these phase inverters is quite apparent. All of the E-fields along the opening of the waveguide are now in-phase and resonate together. Consequently, the phase inverters facilitate the realization of eight collinear, in-phase magnetic dipoles. Hence, high directivity, omnidirectional radiation patterns have been realized as shown in Fig. 4 (b).

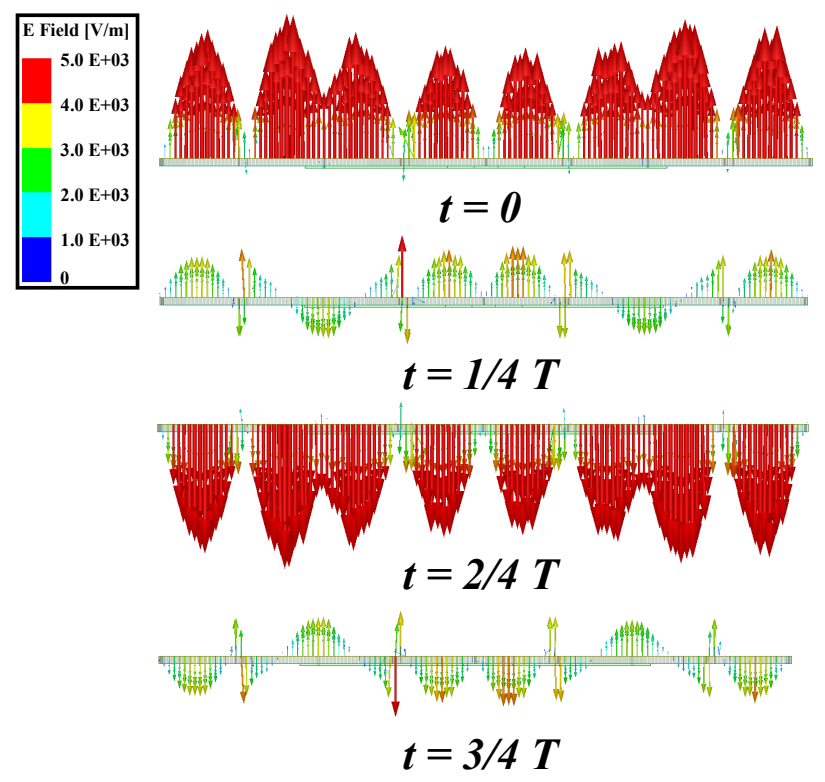

(a)
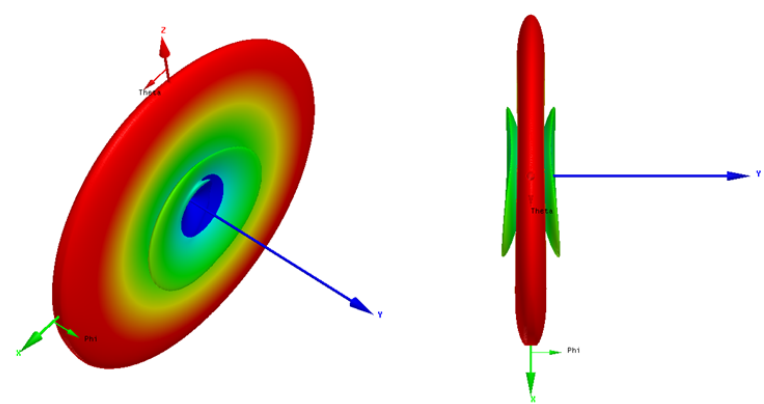

(b)

Figure 4. (a) Electric field distributions along the opening of the waveguide in one period of time T corresponding to the source frequency, $10 \mathrm{GHz}$; and (b) $3 \mathrm{D}$ radiation patterns at $10 \mathrm{GHz}$.

\section{Measurement results}

The fabricated antenna prototype is shown in Fig. 5. It is clear that it is compact. It is very light-weight. The prototype was tested with a Keysight ${ }^{\mathrm{TM}}$ Vector Network Analyzer (VNA) and a MVG far-field compact range system. 


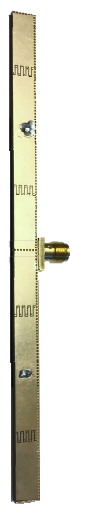

(a)

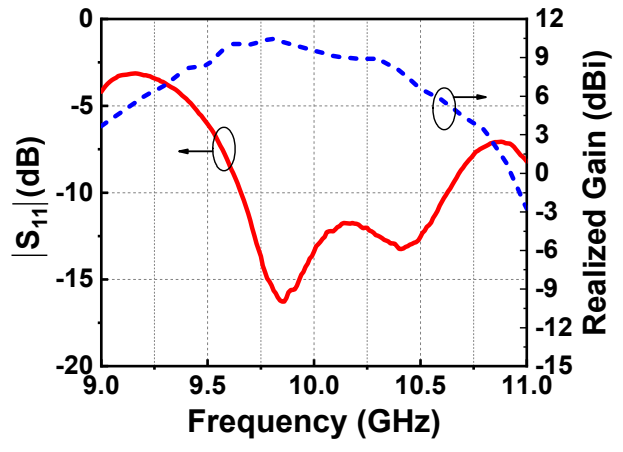

(b)

Figure 5. (a) Fabricated antenna prototype. (b) Measured $\left|S_{11}\right|$ and realized gain values as functions of the source frequency.

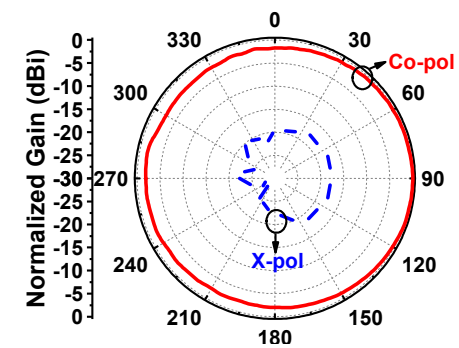

(a)

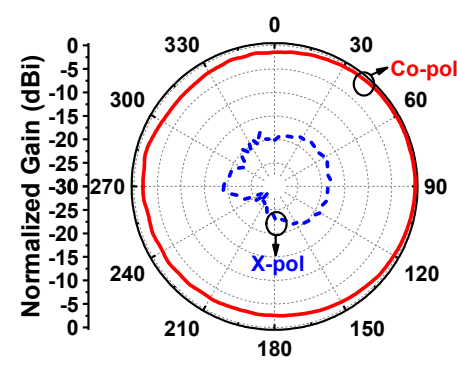

(c)

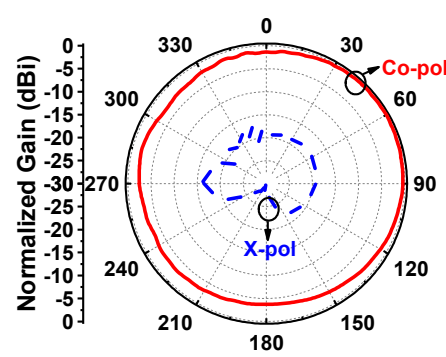

(e)

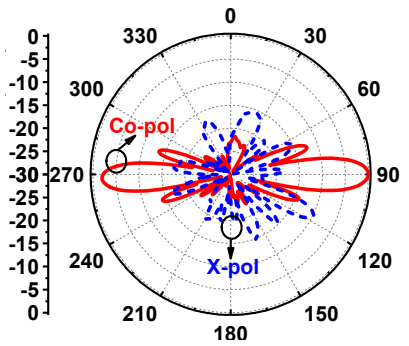

(b)

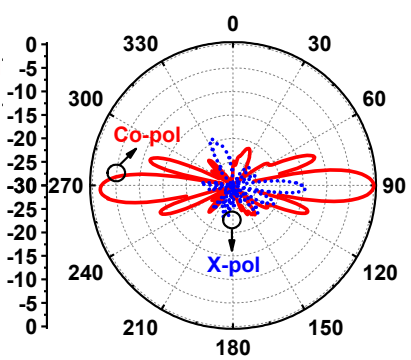

(d)

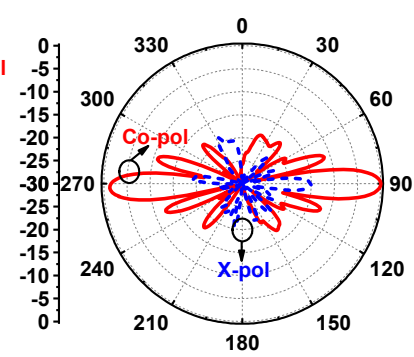

(f)
Figure 6. Measured radiation patterns. (a) Omni-plane at $9.8 \mathrm{GHz}$; (b) Vertical plane at $9.8 \mathrm{GHz}$; (c) Omni-plane at $10 \mathrm{GHz}$; (d) Vertical plane at $10 \mathrm{GHz}$; (e) Omni-plane at $10.2 \mathrm{GHz}$; (f) Vertical plane at $10.2 \mathrm{GHz}$;
The measured $\left|S_{11}\right|$ and realized gain values are shown in Fig. 5. The overlapping $10-\mathrm{dB}$ impedance and $3-\mathrm{dB}$ realized gain bandwidth covers $800 \mathrm{MHz}$ from 9.6 to $10.4 \mathrm{GHz}(8 \%$ fractional bandwidth). The peak realized gain is now $10.4 \mathrm{dBi}$; it achieves a $2.9 \mathrm{~dB}$ gain enhancement in comparison to the system without the phase inverters. The radiation patterns in both the omni-plane (zx, $\varphi=0^{\circ}$ plane) and the horizontal (xy, azimuthal, $\theta=90^{\circ}$ ) plane are shown in Fig. 6. Very good omnidirectional HP radiation performance is observed. The cross polarization level is greater than $17 \mathrm{~dB}$ and the gain variation in the omni-plane is less than $3.0 \mathrm{~dB}$.

\section{CONCLUSIONS}

The basic design, operating principles and measurement results for a high directivity, compact, omnidirectional horizontally polarized (OHP) magnetic dipole antenna array were reported. As will discussed in the presentation, this OHP array has superior performance characteristics (to the best of our knowledge) when compared to all of the previously reported OHP antenna arrays. As will also be demonstrated, this OHP array is an ideal candidate as the power source for wirelessly powering IoT devices such as small battery-less sensors.

\section{REFERENCES}

[1] K.-L. Wong, F.-R. Hsiao, and T.-W. Chiou, "Omnidirectional planar dipole array antenna,” IEEE Trans. Antennas Propag., vol. 52, no. 2, pp. 624-628, Feb. 2004.

[2] Y. M. Pan and K. W. Leung, "Wideband omnidirectional circularly polarized dielectric resonator antenna with parasitic strips," IEEE Trans. Antennas Propag., vol. 60, no. 6, pp. 2992-2997, Jun. 2012.

[3] W. Lin, R. W. Ziolkowski, and T. C. Baum, " $28 \mathrm{GHz}$ compact omnidirectional circularly polarized antenna for Device-to-Device communications in the future 5G systems," IEEE Trans. Antennas Propag., vol. 65, No. 12, pp. 6904-6914, Dec. 2017.

[4] W. Lin and R. W. Ziolkowski, "Compact, high directivity, omnidirectional circularly polarized antenna array," IEEE Trans. Antennas Propag., vol. 67, No. 7, pp. 4537-4547, July 2019.

[5] Y. Li, Z. Zhang, Z. Feng, and M. F. Iskander, "Design of omnidirectional dual-polarized antenna in slender and low-profile column," IEEE Trans. Antennas Propag., vol. 62, no. 4, pp. 2323-2326, Apr. 2014.

[6] J. Wang, L. Zhao, Z. C. Hao, and J.M. Jin, "A wideband dual-polarized omnidirectional antenna for base station/WLAN," IEEE Trans. Antennas Propag., vol. 66, no. 1, pp. 81-87, Jan. 2018.

[7] H. Huang, Y. Liu, and S. Gong, "Broadband dual-polarized omnidirectional antenna for $2 \mathrm{G} / 3 \mathrm{G} / \mathrm{LTE} / \mathrm{WiFi}$ applications," IEEE Antennas Wireless Propag. Lett., vol. 15, pp. 576-579, 2016.

[8] D. Chizhik, J. Ling, and R. A. Valenzuela, "The effect of electric field polarization on indoor propagation," in Proc. IEEE Int. Conf. Universal Pers. Commun. (ICUPC), vol. 1. Oct. 1998, pp. 459-462.

[9] W. Lin, R. W. Ziolkowski and J. Q. Huang, "Electrically small, highly efficient, Huygens dipole rectennas for wirelessly powering Internet-ofThings (IoT) devices," IEEE Trans. Antennas Propag., vol. 67, No. 6, pp. 3670-3679, June 2019.

[10] W. Lin and R. W. Ziolkowski, "Electrically small Huygens CP rectenna with a driven loop element maximizes its wireless power transfer efficiency," IEEE Trans. Antennas Propag., early access, DOI: 10.1109/TAP.2019.2935784.

[11] W. Lin and R. W. Ziolkowski, "Wirelessly powered temperature and light detecting sensors based on electrically small Huygens antennas," Sensors, vol. 19, No. 9, Apr. 2019. 
[12] W. Lin and R. W. Ziolkowski, "Electrically small Huygens antennabased fully-integrated wireless power transfer and communication system," IEEE Access, vol. 7, pp. 39762-39769, Mar. 2019.

[13] Z. Zeng et al., "The design and experiment of a dual-band omnidirectional SIW slot array antenna," in Proc. APMC, Bangkok, Thailand, Dec. 2007, pp. 1-4.

[14] G. Hua, W. Hong, X. H. Sun, and H. X. Zhou, "Design of an omnidirectional line array with SIW longitudinal slot antenna," in Proc. ICMMT, Apr. 2008, pp. 1114-1117.

[15] X. L. Quan, R. L. Li, J. Y. Wang, and Y. H. Cui, "Development of broadband horizontally polarized omnidirectional planar antenna and its array for base stations," Prog. Electromagn. Res., vol. 128, pp. 441-456, Jun. 2012.

[16] Z. Liang, Y. Li, X. Feng, J. Liu, J. Qin, and Y. Long, "Microstrip magnetic monopole and dipole antennas with high directivity and a horizontally polarized omnidirectional pattern," IEEE Trans. Antennas Propag., vol. 66, no. 3, pp. 1143-1152, Mar. 2018.

[17] L. Chang, Y. Li, Z. Zhang and Z. Feng, "Horizontally polarized omnidirectional antenna array using cascaded cavities," IEEE Trans. Antennas Propag., vol. 64, no. 12, pp. 5454-5459, 2016. 